\title{
A face-to-face interview of participants in HUNT 3: the impact of the screening question on headache prevalence
}

\author{
Knut Hagen · John-Anker Zwart • Anne Hege Aamodt $\cdot$ Kristian Berhard Nilsen · \\ Geir Bråthen · Grethe Helde · Marit Stjern · Erling A. Tronvik · Lars Jacob Stovner
}

Received: 12 June 2008/Accepted: 17 July 2008/Published online: 9 August 2008

(C) Springer-Verlag 2008

\begin{abstract}
The aim of this study was to evaluate the effect of the screening question phrasing on the 1-year prevalence figures of headache disorders, including migraine. Of a random sample of 563 invited participants in the NordTrøndelag Health Survey 2006-2008 in Norway, 297 (53\%) met to a face-to-face interview. There were $74.1 \%$ that reported having had headache during the last year, whereas only $31.0 \%$ stated that they had suffered from headache in the same period. The 1-year prevalence of migraine was $17.2 \%$ and of tension-type headache (TTH) $51.9 \%$. Migraine was ten times more likely $(O R=9.96,95 \% \mathrm{CI}$ 4.75-20.91) among those who stated that they were headache sufferers than among those who were not. Only headache sufferers had chronic TTH or medication-overuse headache. Thus "Have you suffered from headache?" can be a useful screening question in population-based questionnaire studies if the goal is to identify most migraineurs and almost all individuals with chronic headache.
\end{abstract}

K. Hagen - J.-A. Zwart - A. H. Aamodt

K. B. Nilsen · G. Bråthen - G. Helde · M. Stjern ·

E. A. Tronvik - L. J. Stovner

Department of Neuroscience, Faculty of medicine,

Norwegian University of Science and Technology,

St Olavs Hospital, 7006 Trondheim, Norway

K. Hagen $(\bowtie)$ A A. H. Aamodt · K. B. Nilsen · G. Bråthen ·

M. Stjern - E. A. Tronvik - L. J. Stovner

Department of Neurology,

Norwegian National Headache Centre,

St Olavs Hospital, 7006 Trondheim, Norway

e-mail: knut.hagen@ntnu.no

J.-A. Zwart

Department of Neurology, Ullevaal University Hospital,

University of Oslo, 0450 Oslo, Norway
Keywords Screening - Question · Headache · Migraine · Prevalence

\section{Introduction}

A careful history taken by a headache specialist is the gold standard for making a valid headache diagnosis. This is, however, time-consuming and costly so therefore only a few large population-based studies have used a face-to-face interview approach by a neurologist.

Most large-scale population-based studies have used telephone interview by lay interviewers, a self-administrated questionnaire, or a combination of a screening questionnaire and an interview by a physician [1]. One or a few screening questions that limit the study population are commonly used [e.g., 2-12]. Validation of the method is always required in epidemiological surveys [1, 13], and it should include evaluation of headache prevalence among screen-negative individuals if a screening question is used. However, only a few studies have reported adjusted headache prevalence figures, taking occurrence of migraine among "screen-negative" into consideration $[5,8]$.

Some population-based questionnaire studies have used a neutral screening question such as "Have you had a headache during the last year?" [e.g., 10], whereas other studies have used a more restricted screening question like "Have you suffered from headache during the last year?" $[2,6,12]$. The latter screening question was used in two consecutive large-scale population-based studies performed between 1995 and 1997 (HUNT 2) [12] and 2006 and 2008 (HUNT 3). In both surveys only individuals who answered "yes" (screen-positive) were asked to fill in the other headache questions. 
In order to validate questionnaire-based information, a personal interview performed by neurologists was performed in a random sample of participants in HUNT 3. Access to questionnaire-data will first be available during 2009.

The aim of this study was to estimate prevalence figures of common headache types, including migraine in a random sample of screen-positive individuals (i.e., those who said "yes" to the question whether they had suffered from headache during the last 12 months) as well as in screennegative (i.e., individuals who answered "no" to this question) in two locations that were part of the HUNT 3 study.

\section{Materials and methods}

The third Nord-Trøndelag health survey (HUNT 3)

All inhabitants aged 20 years or more in Nord-Trøndelag county of Norway ( $n=86,456)$ were invited to participate in the third Nord-Trøndelag Health Survey between October 2006 and June 2008 ("Helseundersøkelsen i NordTrøndelag" = HUNT 3). The county was divided in a total of 25 study areas, and the survey in Verdal was performed from September to November 2007, and in Stjørdal from December 2007 to April 2008. Preliminary data indicate that participation rates in the HUNT 3 study were $52 \%$ of the whole population in Verdal and $50 \%$ in Stjørdal.

Study population the validation study

In the present sub-study of HUNT 3 a random sample of individuals who had participated in HUNT 3 in Verdal and Stjørdal were invited to a face-to-face interview performed by neurologists focusing on four different topics covered in the questionnaire, namely alcohol, sleep, headache and musculoskeletal complaints. All invited had previously answered two different large questionnaires in the HUNT 3 study also covering these topics, but their responses to the questions were unknown for all involved in this study. The main objective of the study was to evaluate the validity of questionnaire-based information which will be analyzed when we get access to the questionnaire data during 2009.

Invitation letters were sent to a random sample on the basis of a list of participants in Verdal and Stjørdal. In HUNT 2 the participation rate was strongly age-dependent, with the highest participation in the age group 60-69, and lowest in the age group 20-29 [14]. To ensure acceptable balanced participation in the present study for both genders in all age groups potential participants were selected from the list of HUNT 3 participants consecutively in the following order; man $\leq 50$ years, man $>50$ years, woman $\leq 50$ years, and woman $>50$ years.
Of the participants in HUNT 3 living in Verdal and Stjørdal a random sample of 563 persons got an invitation letter which included general information about the validation study, also informing that they would be contacted on telephone by our research assistants to give further information and to make an appointment for the personal interview. We got the list of participants in Verdal and Stjørdal very shortly before the time of interview. Hence, due to lack of time, if our research assistants were unable to get in contact despite of several attempts they were instructed to call the next persons on the list. Of the 563 potential participants, we were unable to get in contact with $171(30 \%)$ despite a minimum of two attempts or because no updated or correct telephone number was available. A flow-chart on the selection of participants is given in Fig. 1.

Headache diagnoses

A semi-structured interview was performed by a neurologist with special interest and competence in headache. Initially, all subjects were asked the questions "Have you ever had a headache?", "Have you had a headache during the last 12 months?", and "Have you suffered from headache during the last 12 months?" In the present study individuals who answered "yes" to the latter question "Have you suffered from headache during the last 12 months?" were defined as screen-positive, whereas those who answered "no" were defined as screen-negative. In Norway the question "Have you suffered from headache?" is understood as having had a headache that is quite bothersome.

Individuals who reported headache during the past year were asked about frequency (average number of days per month during the last year), intensity, location, aura symptoms, other migraine and cluster headache features, and use of medication. All diagnoses except for medication overuse headache $(\mathrm{MOH})$ were based on ICHD-II [15], for $\mathrm{MOH}$ the revised version was used [16]. Up to three different headache types were diagnosed in each individual. Subjects with $\mathrm{MOH}$ were also categorized according to their primary headache diagnosis.

\section{Ethics}

The study was included as a part of the HUNT 3 project which was approved by the Regional Committee for Ethics in Medical Research and the Norwegian Data Inspectorate.

Statistics

Demographic data were compared between participants and non-participants with independent sample $t$ test for 
Fig. 1 Diagram of the invited population according to type of participation

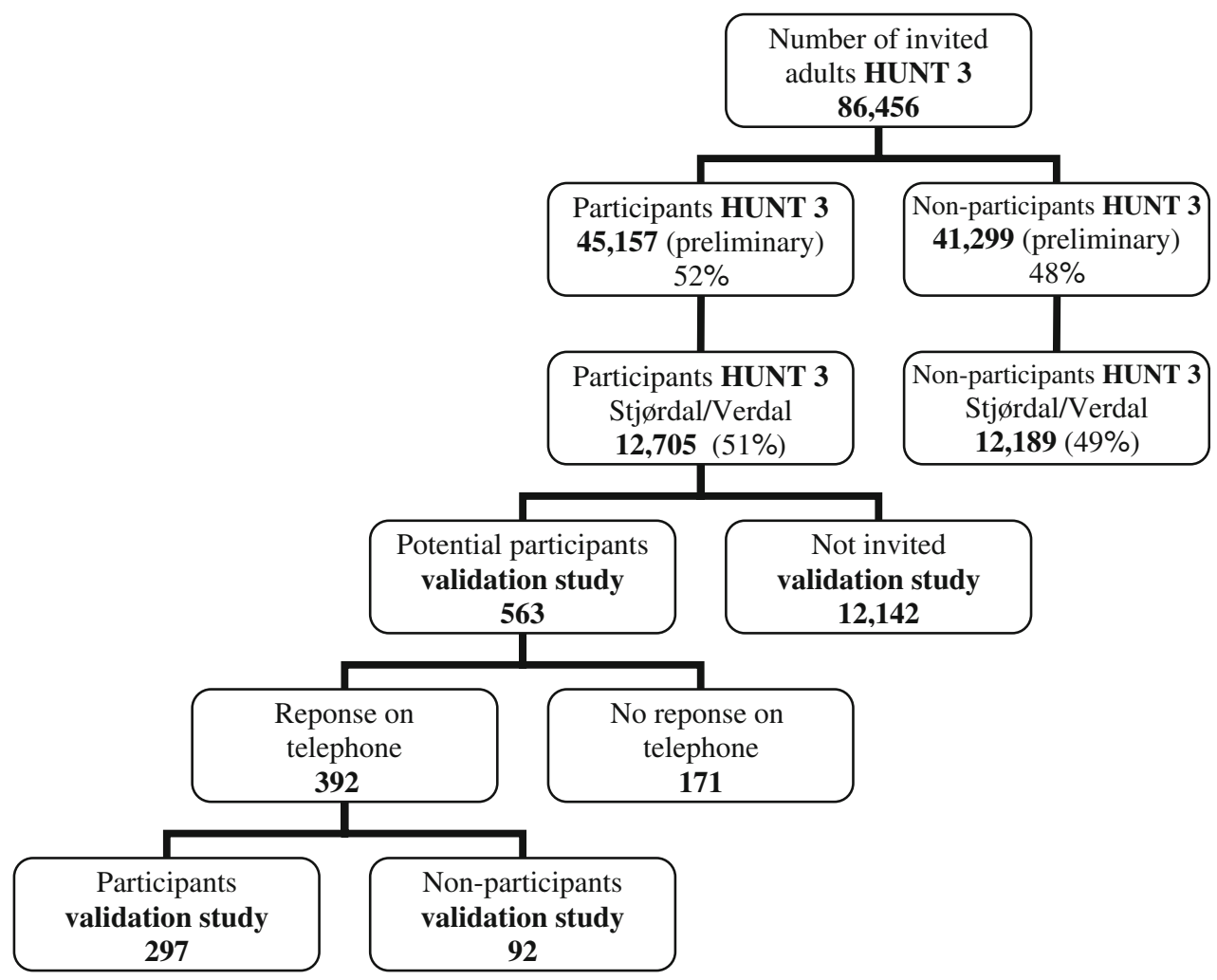

continuous variables and with the chi-squared test for categorical variables. Two-tailed estimations of significance were used, and the level of significance was set at $p<0.05$.

One year prevalence was estimated with $95 \%$ confidence interval (CI). Multivariate logistic regression models were performed to evaluate prevalence odds ratio with $95 \%$ CI among screen-positive, using screen-negative as a reference. Adjustments were performed for age and gender.

Sensitivity, specificity, predictive values (PV), and Cohen's kappa statistics with $95 \%$ CI were calculated for different headache diagnoses among screen-positive, compared to the interview-based diagnoses of the whole sample.

\section{Results}

Of the 563 invited potential participants, we were unable to get in contact by telephone with 171 (Fig. 1). A total of 297 out of the 392 persons we were able to contact by telephone participated (53\% out of the total invited group).

Of the 392 persons who answered on telephone, only 29 stated that they did not want to participate, whereas 66 wanted to participate, but were unable to come because they were out of town, had sick children, were busy in job, or they had forgotten the invitation.
Compared to the 266 non-participants, the 297 participants were older (mean age 52.3 vs. 48.6 years, $p=0.004$ ) and slightly more likely to be men (51 versus $47 \%$, $p=0.37$ ).

Life-time prevalence of not having had headache

Of the 77 subjects who had not had headache during the past year, as much as $40(51.9 \%)$ stated that they never had experienced headache. Hence, the life-time prevalence of not having had headache was $13.5 \%$ (9.6-17.4), the percentage being higher among men (19.1\%, CI 12.8-25.4) than among women $(7.6 \%, 95 \%$ CI 3.2-12.0).

One year prevalence of headache

The 1-year prevalence of different headache disorders by gender from among the 297 participants is shown in Table 1. As demonstrated, $74.1 \%$ (95\% CI 69.1-79.1) reported that they had had headache during the past year, whereas $31.0 \%(25.7-36.3)$ stated that they had suffered from headache (i.e., were screen-positive).

Impact of being screen-positive

Screen-positive headache sufferers were younger (mean age 49.6 vs. 53.5 years, $p=0.028$ ) and more likely to be 
Table 1 One year prevalence of different headache types $(n=297)$. Some participants may have more than one headache diagnosis

\begin{tabular}{|c|c|c|c|}
\hline & $\begin{array}{l}\text { Women } \\
(n=145)\end{array}$ & $\begin{array}{l}\text { Men } \\
(n=152)\end{array}$ & $\begin{array}{l}\text { Overall } \\
(n=297)\end{array}$ \\
\hline Idiopathic stabbing headache $(\%)(95 \% \mathrm{CI})$ & 44.8 (36.6 to 53.0$)$ & 25.7 (18.6 to 32.7$)$ & 35.0 (29.6 to 40.5$)$ \\
\hline Headache, all types (\%) $(95 \% \mathrm{CI})$ & 83.5 (77.3 to 89.6$)$ & $65.1(57.5$ to 72.8$)$ & $74.1(69.1$ to 79.1$)$ \\
\hline Headache sufferer $(\%)(95 \% \mathrm{CI})$ & $37.9(30.0$ to 45.9$)$ & 24.3 (17.4 to 31.2$)$ & $31.0(25.7$ to 36.3$)$ \\
\hline Migraine (\%) (95\% CI) (probable migraine excluded) & $22.1(15.2$ to 28.9$)$ & $12.5(7.2$ to 17.8$)$ & $17.2(12.9$ to 21.5$)$ \\
\hline Migraine with aura $(\%)(95 \% \mathrm{CI})$ & $4.8(1.3$ to 8.4$)$ & $2.0(0.0$ to 4.2$)$ & $3.4(1.3$ to 5.4$)$ \\
\hline Migraine without aura (\%) $(95 \% \mathrm{CI})$ & $14.5(8.7$ to 20.3$)$ & $9.9(5.1$ to 14.7$)$ & $14.5(10.5$ to 18.5$)$ \\
\hline Migraine with or without aura (\%) $(95 \% \mathrm{CI})$ & $2.8(0.1$ to 5.5$)$ & $1.3(0.0$ to 3.2$)$ & $2.0(0.4$ to 3.6$)$ \\
\hline Migraine with coexisting tension-type headache & $11.0(5.9$ to 16.2$)$ & $3.3(0.4$ to 6.2$)$ & $7.1(4.1$ to 10.0$)$ \\
\hline Probable migraine without aura $(\%)(95 \% \mathrm{CI})$ & $2.8(0.1$ to 5.5$)$ & $2.0(0.0$ to 4.2$)$ & $2.4(0.6$ to 4.1$)$ \\
\hline Tension-type headache $(\%)(95 \% \mathrm{CI})$ (probable excluded) & $63.5(55.5$ to 71.4$)$ & 40.8 (32.9 to 48.7$)$ & $51.9(46.1$ to 57.6$)$ \\
\hline Probable tension-type headache $(\%)(95 \% \mathrm{CI})$ & $1.4(0.0$ to 3.3$)$ & $4.0(0.8$ to 7.1$)$ & $2.7(0.8$ to 4.6$)$ \\
\hline Episodic tension-type headache $(\%)(95 \% \mathrm{CI})$ & $57.9(49.8$ to 66.1$)$ & $38.2(30.4$ to 46.0$)$ & $47.8(42.1$ to 53.5$)$ \\
\hline Infrequent episodic TTH (\%) (95\% CI) & 23.5 (16.5 to 30.4 & $21.1(14.5$ to 27.6$)$ & $22.2(17.5$ to 27.0$)$ \\
\hline Frequent TTH (\%) $(95 \%$ CI $)$ & $34.5(26.7$ to 42.3$)$ & 17.1 (11.1 to 23.2$)$ & 25.6 (20.6 to 30.6$)$ \\
\hline Chronic tension-type headache $(\%)(95 \% \mathrm{CI})$ & $4.8(1.3$ to 8.4$)$ & $2.6(0.1$ to 5.2$)$ & $3.7(1.5$ to 5.9$)$ \\
\hline Medication-overuse headache (\%) $(95 \% \mathrm{CI})$ & $1.4(0.0$ to 3.3$)$ & $2.6(0.1$ to 5.2$)$ & $2.0(0.4$ to 3.6$)$ \\
\hline Pure alcohol-induced headache $(\%)(95 \% \mathrm{CI})$ & $0.7(0.0$ to 2.1$)$ & $4.0(0.8$ to 7.1$)$ & $2.4(0.6$ to 4.1$)$ \\
\hline Other headaches $^{\mathrm{a}}(\%)(95 \% \mathrm{CI})$ & $4.1(0.9$ to 7.4$)$ & $2.6(0.1$ to 5.2$)$ & $3.4(1.3$ to 5.4$)$ \\
\hline
\end{tabular}

a Other headaches $(n=10)$ included cervicogenic headache (1 case), caffeine-withdrawal headache (1 case), persistent idiopathic facial pain (1 case), primary exertional headache ( 1 case), headache attributed to rhinosinusitis ( 2 cases) or systemic viral infection (4 cases). One additional case had previously had the diagnosis of cluster headache, but no cluster periods had occurred during the last 4 years

women (59.8 vs. $43.9 \%, p=0.01)$ than screen-negative. The 1-year prevalence of migraine was much higher among screen-positive than among screen-negative (41.3 vs. $6.3 \%, p<0.001)$. The corresponding 1 -year prevalence of tension-type headache (TTH) was $63.0 \%$ and $46.8(p=0.01)$, and of idiopathic stabbing headache 44.5 and $30.7 \%(p=0.02)$. None of the screen-negative had chronic TTH or $\mathrm{MOH}$, giving $100 \%$ specificity and positive PV for these diagnoses among screen-positive (Table 2). As demonstrated by Table 2, the specificity and positive PV increased for migraine and TTH when considering only those with headache $\geq 1$ day per month. The kappa value was moderate for migraine $(0.40,95 \%$ CI $0.27-0.53)$, but poor for TTH $(0.17,0.03-0.25$, Table 2).

In the multivariate analyses, adjusting for age and gender, migraine was ten times more likely $(O R=9.96,95 \%$ CI 4.75-20.91) among screen-positive than among screennegative, whereas TTH $(O R=1.67,0.99-2.83)$ and idiopathic stabbing headache $(O R=1.74,1.02-2.83)$ were both 1.7 times more likely. The difference in prevalence was even more evident for individuals with headache $\geq 1$ days per month: among these migraine was 27 times more likely among screen-positive than screen-negative $(O R=27.9,95 \%$ CI 10.0-78.2) and TTH was six times more likely $(O R=6.5,95 \%$ CI 3.7-11.5).

\section{Discussion}

Using a face-to-face interview by a neurologist the 1-year prevalence of all headache types could be estimated in a randomly selected group of participants in a large-scale population-based survey. Migraine was ten times more likely among screen-positive headache sufferers than among screen-negative, and none of screen-negative had chronic tension-type headache or $\mathrm{MOH}$.

Three out of four answered "yes" to the neutral question "Have you had a headache during the last 12 months" which probably intercepts most headache types. The screening question "Have you suffered from headache during the last 12 months" used in several populationbased studies [e.g., 1, 5, 10] gives much lower headache prevalence, but it may be useful to indentify almost all with chronic headache and most migraineurs, in particular those with a relatively frequent migraine occurring at least 1 day per month. However, this single question is not perfect as an instrument to identify the "true" migraine prevalence since $6.3 \%$ of screen-negative had migraine. A similar finding was done in a Dutch study in which 17 (2\%) out of 863 patients with migraine were identified in the screennegative subsample, and 12 of these had active migraine [8]. The 1-year prevalence estimates for migraine increased from about $12 \%$ when the screen-negative cases were 
Table 2 Sensitivity, specificity, predictive values (PV), and kappa values for different headache diagnoses among screen-positive, compared with the whole sample (screen-positive and screen-negative)

\begin{tabular}{llcccc}
\hline Suffered from headache during the last year $(n=92)$ & Sensitivity & Specificity & Positive (PV) & Negative (PV) & Kappa (95\% CI) \\
\hline Migraine $(n=51)$ & 41 & 94 & 75 & 78 & $0.40(0.27-0.53)$ \\
Migraine $\geq 1$ days per month $(n=42)$ & 40 & 98 & 88 & 78 & $0.44(0.31-0.57)$ \\
Tension-type headache $(n=154)$ & 63 & 53 & 38 & 76 & $0.14(0.03-0.25)$ \\
Tension-type headache $\geq 1$ days per month $(n=88)$ & 59 & 83 & 66 & 82 & $0.43(0.31-0.55)$ \\
Chronic tension-type headache $(n=12)$ & 13 & 100 & 100 & 72 & $0.17(0.01-0.33)$ \\
Medication-overuse headache $(n=6)$ & 7 & 100 & 100 & 70 & $0.09(-0.05-0.25)$ \\
\hline
\end{tabular}

ignored to about $16 \%$ including the screen-negative migraineurs [8]. In a Danish study using "Have you ever had migraine" as a screening question, 15 (11\%) out of 137 who answered "no" in the questionnaire got the diagnosis of migraine by clinical interview. The prevalence of migraine increased from 17.7 to 24.1 when adjustment for migraine prevalence among screen-negative was performed [5].

Our face-to-face interview was performed in a random sample of participants in HUNT 3. The invitation letter did not mention that a detailed headache interview would be performed, but selective participation of headache sufferers may still have occurred. The participation rate was good among individuals we were able to contact by telephone participated, but more moderate (53\%) when the total invited group was considered. Whether there has been a selective participation based on headache status will become clearer when information about headache status among non-participants becomes available during 2009. The overall preliminary participation rate of the questionnaire-based survey in the study area was estimated to be about $51 \%$. Because the overall participation rate of this study was only $27 \%$ when the total invited group in HUNT 3 in Verdal/Stjørdal was considered, generalization of our results to the whole population must be done with caution.

We estimated 1-year prevalence from among the 297 individuals who met at the interview, but these figures have to be adjusted if headache status among participants differs compared to non-participants. The 1-year migraine prevalence of $17.2 \%$ in the present study is somewhat higher than the 12\% found in HUNT 2 performed 1995-1997 [10]. If none of the non-participants $(n=95)$ had migraine, the 1-year prevalence would have to be adjusted to $13.0 \%$, which should be considered as a minimum estimate. In addition to potential selective participation of the present study, the observed increase in prevalence of migraine may be due to differences in study design (questionnaire vs. face-to-face interview), or to a real change in migraine prevalence over a 11-year period.

Relatively, few headache epidemiological studies have used face-to-face interviews by neurologists in the general population. Our 1-year prevalence of migraine of $17.2 \%$ is somewhat higher than the 11 and $15.5 \%$ reported in the two studies from Copenhagen [17, 18], but quite similar (13.0\%) if we take all the 392 invited persons into account. However, whichever way one calculates, the 1-year prevalence of TTH in the present study of $51.9 \%$ was lower than the prevailing 79 and $87 \%$ reported in the two Copenhagen studies [17, 18]. Interestingly, our 1-year prevalence of idiopathic stabbing headache of 35\% was identical to the prevalence in Vågå [19] (35.2\%) in Norway a few years ago. Regarding chronic headache the 1-year prevalence of $\mathrm{MOH}$ (2.0\%) and chronic TTH (3.7\%) were slightly higher than the 1.7 and $2.8 \%$ (probable chronic TTH included) recently reported in Akershus in Norway among 30- to 44-year-old persons [10, 20]. Our study also included older age groups, which most likely explain the somewhat higher prevalence of chronic headache.

The value of having only a few questions as a screening tool is emphasized for headache as well as other disorders [e.g., 1, 21, 22]. Which screening questions would be optimal in headache epidemiological studies? Theoretically, a neutral question would have the highest sensitivity to detect all cases. Additional questions on headache frequency and severity could then be used to define groups of clinical and economical interest [23]. However, a more selective screening question may be preferable in large questionnaire-based survey like the HUNT study where headache questions constitute a minor part of a large questionnaire, in order to minimize the rate of incomplete answers. A combination of a screening question and a clinical interview by a physician has been recommended as a cost-effective method to conduct an epidemiological survey on chronic headache [1].

Asking whether they had suffered from headache during the last year gave high specificity and high positive predictive value for identifying individuals with $\mathrm{MOH}$, chronic TTH, and migraine $\geq 1$ day/month. The moderate kappa value for migraine indicated that this single question is not optimal as a screening tool to identify all migraineurs in the population. When a screening questionnaire is used, answers to a combination of several questions will increase 
the chance-corrected agreement rate (kappa value), as illustrated in the validation study of HUNT 2 [24]. Selfreported migraine alone had a kappa value of 0.43 , which increased to 0.59 by using answers of a combination of questions [24].

Acknowledgments The Nord-Trøndelag Health Study (The HUNT study) is a collaboration between The HUNT Research Centre, Faculty of Medicine, The Norwegian University of Science and Technology (NTNU), and the Nord-Trøndelag County Council.

\section{References}

1. Russell MB, Aaseth K, Grande RB, Gulbrandsen P, Lundqvist C (2007) Which strategy should be applied? Design of a Norwegian epidemiological survey on chronic headache. Acta Neurol Scand 115(187):59-63

2. D'Alessandro R, Benassi G, Lenzi PL, Gamberini G, Sacquegna T, De Carolis P, Lugraesi E (1988) Epidemiology of headache in the republic of San Marino. J Neurol Neurosurg Psychiatry 51:21-27

3. Henry P, Michel P, Brochet B, Dartigues JF, Tison S, Salamon R et al (1992) A nationwide survey of migraine in France: prevalence and clinical features in adult. Cephalalgia 12:229-237

4. Stewart WF, Lipton RB, Celentano DD, Reed ML (1992) Prevalence of migraine headache in the United States. Relation to age, race, and other sociodemographic factors. JAMA 267:64-69

5. Russell MB, Rasmussen BK, Thorvaldsen P, Olesen J (1995) Prevalence and sex-ratio of the subtypes of migraine. Int J Epidemiol 24:612-618

6. Mitsikostas DD, Tsaklakidou D, Athanasiadis N, Thomas A (1996) The prevalence of headache in Greece: correlations to Latitude and climatological factors. Headache 36:168-173

7. Castillo J, Muñoz P, Guitera V, Pascual J (1999) Epidemiology of chronic daily headache in the general population. Headache 39:190-196

8. Launer LJ, Terwindt GM, Ferrari MD (1999) The prevalence and characteristics of migraine in a population-based cohort: the GEM study. Neurology 53:537-542

9. Russell MB, Levi N, Saltyte-Benth J, Fenger K (2006) Tensiontype headache in adolescents and adults: a population based study of 33, 764 twins. Eur J Epidemiol 21:153-160

10. Grande RB, Aaseth K, Gulbransen P, Lundquist C, Russell MB (2008) Prevalence of primary chronic headache in a population- based sample of 30- to 44-year-old persons. The Akershus study of chronic headache. Neuroepidemiology 30:76-83

11. Nikiforow R (1981) Headache in a random sample of 200 persons: a clinical study of a population in northern Finland. Cephalalgia 1:99-107

12. Hagen K, Zwart JA, Vatten L, Stovner LJ, Bovim G (2000) Prevalence of migraine and non-migrainous headache-HeadHUNT, a large population-based study. Cephalalgia 20:900-906

13. Hagen K, Stovner LJ, Zwart JA (2007) Potentials and pitfalls in analytical headache epidemiological studies: lessons to be learned from the Head-HUNT Study. Cephalalgia 27:403-413

14. Holmen J, Midthjell K, Krüger $\varnothing$, Langhammer A, Holmen TL, Bratberg GH, Vatten L, Lund-Larsen PG (2003) The NordTrøndelag Health study 1995-1997 (HUNT 2): objectives, contents, methods and participation. Nor J Epidemiol 13:19-32

15. Headache classification committee of international headache society (2004) The international classification of headache disorders. 2nd edn. Cephalalgia 24(1):88-101

16. Olesen J, Bousser MG, Diener HC, Dodck D, First M, Goadsby PJ, Göbel H, Lainez MJA, Lance JW, Lipton RB, Nappi G, Sakai F, Schoenen J, Silbertstein SD, Steiner TJ (2006) New appendix criteria open for a broader concept of chronic migraine. Cephalalgia 26:742-746 (Headache classification committee)

17. Rasmussen BK, Jensen R, Schroll M, Olesen J (1991) Epidemiology of headache in a general population: a prevalence study. $\mathrm{J}$ Clin Epideiol 44:1147-1157

18. Lyngberg AC, Rasmussen BK, Jorgensen T, Jensen R (2005) Has the prevalence of migraine and tension-type headache changed over a 12-year period? A Danish population survey. Eur J Epidemiol 20:243-249

19. The Vågå study, epidemiology of headache (2001) The prevalence of ultrashort paroxysms. Cephalalgia 21:207-215

20. Aaseth K, Grande RB, Kværner KJ, Gulbransen P, Lundquist C, Russell MB (2008) Prevalence of secondary chronic headache in a population-based sample of 30- to 44-year-old persons. The Akershus study of chronic headache. Cephalalgia 28:705-713

21. Mitchell AJ (2008) Are one or two simple questions sufficient to detect depression in cancer and palliative care? A Bayesian metaanalysis. Br J Cancer (in press)

22. Jinks C, Lewis M, Ong BN, Croft P (2001) A brief screening tool for knee pain in primary care. 1 . Validity and reliability. Rheumatology 40:528-536

23. Stovner LJ, Zwart JA, Hagen K, Terwindt GM, Pascual J (2006) Epidemiology of headache in Europe. Eur J Neurol 13:333-345

24. Hagen K, Zwart JA, Vatten L, Stovner LJ, Bovim G (2000) HeadHUNT: validity and reliability of a headache questionnaire in a large population-based study in Norway. Cephalalgia 20:244-251 